\title{
Riesgos y beneficios de la suplementación con calcio y con vitamina D
}

\author{
Benefits and risks of calcium and D vitamin supplementation
}

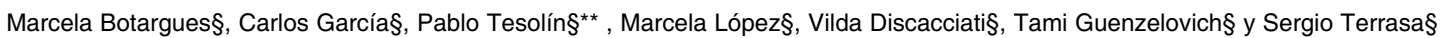

\begin{abstract}
Resumen
Los suplementos de calcio y/o vitamina $D$ son muy utilizados para la prevención y el tratamiento de la osteoporosis. Diariamente atendemos pacientes que desean suspenderlos por intolerancia digestiva o, simplemente, no quieren iniciarlos; y por otro lado, tenemos dudas respecto de su efectividad y sus potenciales riesgos.

En ese contexto se planteó en el Servicio de Medicina Familiar y Comunitaria del Hospital Italiano una actividad de discusión en la que fueron presentados cuatro trabajos recientemente publicados (dos investigaciones, un consenso de expertos y una revisión narrativa), con el objetivo de discutir cuál es la evidencia actual para recomendar los suplementos de calcio para la prevención de fracturas y su potencial asociación con un aumento del riesgo de enfermedad cardiovascular.

En este artículo se describen primero los resúmenes de las cuatro publicaciones presentadas, y luego se ofrece una síntesis y un comentario de la discusión.

\section{Abstract}

Calcium supplements and or vitamin D are widely used for prevention and treatment of osteoporosis. We daily attend patients who wish to suspend them owed to digestive intolerance or wish simply not to start, and on the other hand, we have doubts about its effectiveness and its potential risks.

In this context in the Department of Family and Community Medicine at the Hospital Italiano raised a discussion activity in which four recently published papers were described (two original investigations, a consensus of experts and a narrative review), in order to discuss wich is the current evidence to recommend calcium supplements to prevent fractures and their potential association with an increased risk of cardiovascular disease.

This article first describes a summary of those four publications, and then offers a synthesis of the discussion.
\end{abstract}

\begin{abstract}
Palabras clave: suplementación con calcio, suplementación con vitamina D, riesgo cardiovascular, prevención de fracturas. Key words: calcium sup-
\end{abstract} plementation, D vitamin supplementation, cardiovascular risk, fractures prevention.

Botargues M, García C, Tesolín P, López M, Guenzelovich T y Terrasa S. Riesgos y beneficios de la suplementación con calcio y con vitamina. Evid Act Pract Ambul. Jul-Sep 2011;14(3): 100-102.

\begin{abstract}
1)Asociación entre la magnitud del consumo de calcio a largo plazo y el riesgo de fractura y de osteoporosis (síntesis de una cohorte')
\end{abstract}

Warensjo y col. BMJ 2011;342: d1473.

\section{Objetivos}

Investigar la asociación entre la magnitud del consumo de calcio a largo plazo y el riesgo de fractura.

\section{Diseño}

Estudio longitudinal prospectivo de cohortes, llevado a cabo a nivel poblacional en dos ciudades de Suecia. Participaron 61.433 mujeres (nacidas entre 1914 y 1948) que fueron seguidas durante 19 años. La cohorte original invitó a las participantes a realizar periódicamente un rastreo mamográfico, determinó la incidencia de fracturas y evaluó periódicamente la dieta de las mujeres a través de cuestionarios, clasificándolas en cinco quintilos de consumo diario de calcio: menos de $751 \mathrm{mg} ; 751$ a 882,882 a 996, 996 a 1.137 y más de 1.137 . Además, 5.022 de las participantes conformaron una subcohorte en donde se realizó un seguimiento densitométrico.

\section{Medición de los resultados principales}

Incidencia de fracturas de cualquier tipo, fracturas de cadera (resultados primarios) y de osteoporosis (resultado secundario).

\section{Resultados}

14.738 mujeres (24\%) tuvieron una primera fractura de cualquier tipo y de estas, $3.871(6 \%)$ fueron de cadera. De la subcohorte de 5.022 mujeres, 1.012 (20\%) desarrollaron osteoporosis.

Los patrones de riesgo con el consumo de calcio no fueron lineales. Por ejemplo, la tasa cruda de primera fractura fue de $17,2 / 1.000$ personas años en el quintilo más bajo de consumo de calcio y de 14,0/1.000 personas años en el tercer quintilo, con un Hazard ratio* multivariado 1,18 (IC95\% 1,12 a 1,25). El HR para una primera fractura de cadera fue de $1,29(1,17 \mathrm{a}$ $1,443)$ y el OR para osteoporosis de 1,47 (1,09 a 2).

Por otro lado, estar en el quintilo más alto de consumo de calcio no se asocio a un menor riesgo de fracturas u osteoporosis pero si a una mayor tasa de fracturas de cadera (HR 1,19; 1,06 a 1,32).

\section{Conclusiones}

El incremento gradual del consumo de calcio por encima del nivel del segundo quintilo no se asoció con mayores reducciones en el riesgo de fractura u osteoporosis. Debe ponerse énfasis en individuos con bajo consumo de calcio más que en incrementar el consumo en quienes ya consumen cantidades satisfactorias.

2) Evaluación, tratamiento y prevención del déficit de Vitamina D (síntesis de un consenso de expertos²)

Holick M y col. J Clin Endocrinol Metab 2011;96:1911-1930.

\section{Objetivo}

Proporcionar directrices para la evaluación, tratamiento y prevención de la deficiencia de vitamina $D$ con énfasis en el cuidado de los pacientes que están en riesgo de padecerla.

\section{Participantes}

El grupo de trabajo estuvo compuesto por un presidente, seis expertos adicionales y un metodólogo, que no recibieron financiamiento.

\section{Proceso de consenso y sistema de reporte}

Se basó en una revisión sistemática de la evidencia y discusiones presenciales y por correo electrónico entre los participantes del panel. El proyecto preparado por el Grupo de

\footnotetext{
$\S$ Servicio de Medicina Familiar y Comunitaria del Hospital Italiano de Buenos Aires. maria.botargues@hospitalitaliano.org.ar
}

** Instituto Universitario del Hospital Italiano de Buenos Aires. 
Trabajo fue revisado sucesivamente por el Subcomité Clínico y por el Comité de los Asuntos Clínicos Principales de la Sociedad de Endocrinología de EE.UU. (SE) y por las otras asociaciones co-patrocinadores. Fue publicado en el sitio web de la SE para ser revisado por sus miembros en todas las etapas del proceso, recibiendo el grupo de trabajo observaciones por escrito e incorporando los cambios necesarios. Se utilizó la clasificación "GRADE" de las recomendaciones de acuerdo al grado de evidencia que las sustenta.

\section{Conclusiones}

Teniendo en cuenta que la deficiencia de vitamina $D$ es muy común en todas las edades y que pocos alimentos la contienen, el Grupo de Trabajo recomienda su suplementación diaria, tendiendo a que el individuo ingiera cantidades cercanas a las que proveen niveles cercanos al máximo tolerable diario, de acuerdo a su edad y sus circunstancias clínicas.

El Grupo de Trabajo también sugirió la medición de los niveles de 25-hidroxivitamina $\mathrm{D}$ mediante alguna prueba confiable en los individuos con mayor riesgo de presentar deficiencia (embarazadas y mujeres que amamantan, adultos mayores y con historias de caídas y fracturas no traumáticas, niños obesos y adultos obesos con un índice de masa corporal mayor a $30 \mathrm{~kg} / \mathrm{m} 2$, pacientes con osteoporosis, insuficiencia renal crónica, osteomalacia, falla hepática, síndromes de malabsorción, fibrosis quística, enfermedad inflamatoria intestinal, enteritis por radiación, hiperparatiroidismo, sarcoidosis, tuberculosis, histoplasmosis, coccidiomicosis y linfomas, en quienes han sido operados de una cirugía bariátrica, y en quienes usan medicaciones anticonvulsivantes y antiretrovirales, glucocorticoides, antifúngicos como ketoconazol, colestiramina), recomendando el tratamiento con vitamina D2 o D3 en quienes se documente dicha deficiencia.

Concluyó además que en la actualidad todavía no existe evidencia suficiente como para recomendar el rastreo de niveles de vitamina $D$ en personas que no poseen alto riesgo de tener deficiencia de la misma, o de prescribir dicha vitamina con el objetivo de prevenir la enfermedad cardiovascular.

3) Asociación entre la suplementación de calcio o de calcio más vitamina $\mathrm{D}$ y la incidencia de eventos cardiovasculares (síntesis de un metanálisis ${ }^{3}$ )

Bolland y col. BMJ 2011; 342:d2040.

\section{Objetivos}

Investigar el efecto de los suplementos de calcio sobre el riesgo cardiovascular.

\section{Métodología}

Reanálisis de los datos del ensayo clínico "Iniciativa de salud de las mujeres, calcio/vitamina D" (en inglés: "Women Health Initiative /CaD study") y posterior metanálisis de sus datos junto a otros estudios. El WHI/Cad fue un ensayo clínico aleatorizado de siete años de seguimiento que había incluido a 36.282 mujeres de la comunidad y las había asignado a dos ramas de tratamiento: 1) $1 \mathrm{~g}$ de calcio más $400 \mathrm{UI}$ de vitamina $\mathrm{D}$; 2) placebo.

\section{Medición de resultados principales}

Incidencia de cuatro eventos cardiovasculares y sus combinaciones (infarto agudo de miocardio [IAM], revascularización coronaria, muerte por enfermedad coronaria y accidente cerebrovascular).

\section{Resultados}

En el subgrupo de mujeres que no estaban tomando suplementos de calcio antes de la aleatorización, la incorporación de los mismos consecutivamente a ser incluidas en la rama intervención se asoció a una tendencia sin significancia estadística en el aumento de la incidencia de eventos cardiovasculares (HR 1,13 ; IC $95 \% 0,99$ a 1,29 para el evento combinado con similar significancia estadística para cada uno de los eventos analizados por separado); sin observarse esta tendencia en las que ya venían consumiendo suplementos.

Cuando se meta-analizaron estos resultados junto con los de otros dos ensayos clínicos ${ }^{5,6}$ que habían comparado la suplementación de calcio más vitamina $\mathrm{D}$ contra placebo (llegándose a un número total de participantes de 20.090), dicha suplementación se asoció a un aumento del riesgo de infarto agudo de miocardio (RR 1,21; IC95\% 1,01 a 1,44) y accidente cerebrovascular (RR 1,2; IC95\% 1 a 1,43).

Un posterior meta-análisis que incluyó los datos de nueve ensayos clínicos $^{3,4,5,7,8,9,10,11,12,13}(\mathrm{n}=28.072$ participantes) mostró que la suplementación de calcio o de calcio más vitamina $D$ se asoció a un aumento en el riesgo de IAM (RR 1,24; IC95\% 1,07 a 1,45).

\section{Conclusiones}

La suplementación de calcio y la suplementación de calcio más vitamina $D$ se asocia a modestos incrementos de la incidencia de eventos cardiovasculares.

4) Evidencia complementaria respecto de la suplementación de calcio y/o vitamina $D$ respecto del riesgo cardiovascular (síntesis de una revisión narrativa4)

Rosen H. UpToDate 19.2.

Más allá del reporte del metanálisis resumido en el punto 3 , la evidencia respecto del efecto de la suplementación de calcio sobre el riesgo cardiovascular, es controvertida. Por ejemplo, otro metanálisis del mismo autor ${ }^{15}$ que había evaluado el efecto de la suplementación con calcio mas vitamina $D$ también arrojó resultados preocupantes respecto de un posible aumento del riesgo de IAM en los asignados a suplementación con calcio vs. placebo (RR: 1,27; IC95\%: 1,01 a 1,59). Sin embargo, esta información debe tomarse con cuidado ya que como estos ensayos clínicos no habían sido diseñados para explorar resultados cardiovasculares, los autores de dicha revisión sistemática no contaron con los datos completos como para sacar conclusiones; y además la ingesta basal de calcio en la dieta había variado desde 750 hasta $1240 \mathrm{mg}$ diarios, y la adición de suplementos había implicado una ingesta total entre 1500 y $2000 \mathrm{mg}$ diarios (superior a la recomendada actualmente).

Otro metanálisis ${ }^{16}$ evaluó los efectos de los suplementos de calcio, vitamina $\mathrm{D}$ o ambos, en la incidencia de enfermedad cardiovascular (ECV), con varios puntos finales medidos (muerte, enfermedad coronaria no fatal, IAM y accidente cerebrovascular no fatal). En un análisis combinado de cuatro estudios, la suplementación con calcio no mostró aumentar significativamente el riesgo de eventos cardiovasculares cuando se lo comparó con placebo (RR 1,14; IC95\% 0,92 a 1,41).

\section{Comentario}

La recomendación clásica es que las mujeres consuman durante su postmenopausia $1200 \mathrm{mg} /$ día de calcio en su dieta más $800 \mathrm{Ul}$ de vitamina $\mathrm{D}^{17}$. Cantidades inferiores de vitamina $\mathrm{D}^{\dagger+}$ no serían eficaces, mientras que altas dosis durante largos periodos podrían ser tóxicas. Vale destacar que la leche es la 
mejor fuente dietética de vitamina $\mathrm{D}$, con aproximadamente 100 UI por taza, y que no está consensuada la ingesta recomendada de calcio y vitamina $\mathrm{D}$ en mujeres pre-menopáusicas ni en hombres con osteoporosis.

Respecto del calcio, los suplementos son económicos y están recomendados en individuos cuyo aporte en la dieta es considerado insuficiente y en pacientes con diagnóstico de osteoporosis (evidencia grado $2 \mathrm{~b})^{\ddagger}$. Sin embargo, es importante tener en cuenta que la ingesta total de calcio (dieta más suplementos) no debe exceder los 2000 mg diarios, siendo el límite máximo seguro de vitamina D 4000 UI por día.

La evidencia que respalda el beneficio de la suplementación de calcio más vitamina $D$ en pacientes con osteoporosis proviene de estudios prospectivos, aleatorizados y controlados con placebo. Por ejemplo, un metanálisis (comentado en este número de EVIDENCIA) de doce ensayos aleatorizados $(n=42.279$ pacientes con un promedio de 78 años de edad) informó que la suplementación con vitamina D (700 a 800 unidades) se asoció a un menor riesgo relativo de fracturas no vertebrales (RR 0,80 IC 95\% 0,72 a 0,89) y de cadera $(0,82$ IC $95 \% 0,69$ a 0,97$)$; sin haberse podido observar beneficios significativos con dosis bajas de vitamina D (400 UI/día).

Otra revisión sistemática ${ }^{19}$ revisó 29 ensayos aleatorizados $(n=63.892)$ que habían incluido personas de más de 50 años. Del metanálisis de los 17 ensayos clínicos $(n=52.625)$ que habían analizado como resultado principal a la reducción de la incidencia de fracturas (el resto sólo había contemplado variables densitométricas) pudo evidenciarse que la suplementación con calcio más vitamina $D$ se asoció a una reducción de la incidencia de fracturas de 12\% (RR 0,88; IC95\% 0,83 a 0,95$)$.

Dado que el déficit de vitamina $D$ es muy frecuente en todos los grupos de edad y que son pocas las fuentes alimentarias que la proveen, como resumimos previamente, el consenso de expertos de la Sociedad de Endocrinología de EE.UU..$^{13}$ se expidió a favor del rastreo de su deficiencia en individuos en riesgo de padecerla y su eventual suplementación cuando su concentración sérica sea menor a $30 \mathrm{ng} / \mathrm{dL}$, con el objetivo de mejorar la masa muscular, prevenir caídas y fracturas. Sin embargo, durante la discusión se le criticaron varios aspectos a este consenso: 1) su escasa representatividad por no haber incluido en el grupo de trabajo que confeccionó el documento a miembros de otras especialidades, por ejemplo las de atención primaria como médicos de familia o clínicos y menos aún pacientes; 2) no haber establecido objetivos bien definidos; 3) haberse basado en evidencia con poco énfasis en resultados duros como, por ejemplo, incidencia de fracturas.

\section{Conclusiones de la discusión}

Si bien hay trabajos que demostraron que los suplementos de calcio y/o vitamina $D$ prevendrían las fracturas, los grandes estudios aleatorizados todavía no han podido demostrarlo, no existiendo tampoco todavía datos contundentes respecto del supuesto aumento de la incidencia de eventos cardiovasculares asociado a dicha suplementación.

Recordemos que la osteoporosis es uno de los factores de riesgo de fracturas y no una enfermedad en sí misma, y que además las fracturas se asocian no solo a la osteoporosis (como ha logrado instalar en la opinión pública la industria farmacéutica) sino al complejo y multifactorial fenómeno de las caídas, que se comportan como un suceso multicausal. En la génesis de las caídas intervienen la falta de masa, reflejos, y/o tono muscular (asociados a su vez a déficits nutricionales y de actividad física que conducen a la sarcopenia, al consumo de medicaciones depresoras del sistema nervioso central) a cuestiones ambientales como las vinculadas a la seguridad en el hogar (ej. iluminación, obstáculos en los pasillos, etc.) o en las veredas, al miedo posterior luego de una caída previa, etc. Por lo tanto, un fenómeno complejo como las caídas requiere soluciones complejas que van más allá de la prescripción de suplementos para fortalecer los huesos. En este número de Evidencia se discute una revisión de la Fuerza de Tareas Preventiva Norteamericana (USPSTF) que aborda la eficacia de las principales intervenciones destinadas a reducir las caídas en adultos mayores ${ }^{20}$.

Vale destacar, además, que los suplementos que contienen calcio suelen ser mal tolerados (provocan distensión abdominal y constipación), lo que conduce a que los pacientes tengan baja adherencia a nuestras indicaciones; los usen sólo en forma intermitente o los abandonen luego de unos meses de que les fueron prescriptos. Dada esta situación consideramos que promoviendo el incremento del consumo de calcio en la dieta alimentaria, estaremos menos compelidos a indicarlo en forma suplementada.

Referencias

1. Warensjo y col. Dietary calcium intake and risk of fracture and osteoporosis: prospective longitudinal cohort study. BMJ 2011;342: d1473.

2. Holick M y col. Evaluation, Treatment, and Prevention of Vitamin D Deficiency: an Endocrine Society Clinical Practice Guideline. J Clin Endocrinol Metab 96: 1911-1930, 2011.

3. Bolland M y col. Calcium supplements with or without vitamin D and risk of cardiovascular events: reanalysis of the Women's Health Initiative limited access dataset and meta-analysis. BMJ 2011; 342:d2040

4. Bolland $\mathrm{M}$ y col. Vascular events in healthy older women receiving calcium supplementation: randomised controlled trial. BMJ 2008;336:262-6.

5. Grant A y col. Oral vitamin D3 and calcium for secondary prevention of low-trauma fractures in elderly people (Randomised Evaluation of CalciumOr vitamin D: RECORD): a randomised placebo-controlled trial. Lancet 2005;365:1621-8.

6. Lappe J y col. Vitamin D and calcium supplementation reduces cancer risk: results of a randomized trial. Am J Clin Nutr 2007:85:1586-91

7. Reid I y col. Effect of calcium supplementation on bone loss in postmenopausal women. NEnglJ Med 1993;328:460-4.

8. Reid I y col. Long-term effects of calcium supplementation in postme loss and fractures in postmenopausal women: a randomized controlled trial. Am J Med 1995:98:331-5

9. Baron J y col. Calcium supplements for the prevention of colorectal adenomas. Calcium Polyp Prevention Study Group. NEnglJMed 1999;340:101-7.

9. Baron J y col. Calcium supplements for the prevention of colorectal adenomas. Cals
10. Reid I y col. Randomized controlled trial of calcium in healthy older women. Am J

10. Reid I y col. Rando

Med 2006;119:777-85.

11. Reid I y col. Randomized controlled trial of calcium supplementation in healthy, non-osteoporotic, older men. Arch Intern Med 2008;168:2276-82.

12. Prince R y col. Effects of calcium supplementation on clinical fracture and bone structure: results of a 5-year, double-blind, placebo-controlled trial in elderly women. Arch Intern Med 2006;166:869-75

13. Bonnick y col. Treatment with alendronate plus calcium, alendronate alone, or calcium alone for postmenopausal low bone mineral density. Curr Med Res Opin 2007;23:1341-9.

14. Rosen H. Calcium and vitamin D supplementation in osteoporosis. En: Mulder J edior. UpToDate 19.2. Last updates 05/2011. Disponible en URL: www.uptodate.com (último acceso: 01/08/11).

15. Bolland $\mathrm{M}$ y col. Effect of calcium supplements on risk of myocardial infarction and cardiovascular events: meta-analysis. BMJ. 2010;341:c3691.

16. Wang L y col. Systematic review: Vitamin D and calcium supplementation in prevention of cardiovascular events. Ann Intern Med. 2010;152(5):315

17. Rosen H. Calcium and vitamin D supplementation in osteoporosis. En: Mulder J editor. UpToDate 19.2. Disponible en URL bajo suscripción: http://www.uptodate.com/contents/calcium-and-vitamind-supplementation-in-osteoporosis?source=search_result\&selectedTitle=3\%7E150 (último acceso 05/08/11).

18. Galich AM. La vitamina D oral es efectiva para la prevención de fracturas no vertebrales en individuos de 65 años o más. Evid Act Pract Ambul. Jul-Set 2011;14(3):xx. Comentado de: Bischoff-Ferrari HA Willett WC Wong JB y col. Prevention of Nonvertebral Fractures With Oral Vitamin D and Dose Dependency. A Meta-analysis of Randomized Controlled Trials. Arch Intern Med 2009;169(6):551561. PMID: 19307517

19. Tang B y col. Use of calcium or calcium in combination with vitamin D supplementation to prevent fractures and bone loss in people aged 50 years and older: a meta-analysis. Lancet. 2007;370(9588):657.

20. Giber F. Prevención de caídas en ancianos: revisión sistemática de la evidencia de la Fuerza de Tareas Preventiva de los EE.UU. Evid Act Pract Ambul. 2011;14(3):XX. Comentado de: Michael Y, Whitlock E, Lin J, y col. Primary Care-Relevant Interventions to Prevent Falling in Older Adults: A Systematic Evidence Review for the U.S. Preventive Services Task Force. Ann Intern Med. Dec 20, 2010;153(12):815-825. PMID: 21173416. 